(2) Open Access Full Text Article

\title{
Intrasound therapy in tendon healing: is intensity
}

\section{a factor?}

This article was published in the following Dove Press journal:

Open Access Rheumatology: Research and Reviews

4 August 2010

Number of times this article has been viewed

\section{Al Aiyegbusi' \\ FIO Duru' \\ SR Akinbo ${ }^{2}$ \\ CC Noronha' \\ AO Okanlawon'}

'Departments of Anatomy and

${ }^{2}$ Physiotherapy, College of Medicine,

University of Lagos, Lagos, Nigeria
Correspondence: Al Aiyegbusi

Department of Anatomy,

College of Medicine, University

of Lagos, Lagos, Nigeria

Mobile +2348023212513

Email bogphysio@yahoo.com
Objective: This study investigated the effects of low- and high-intensity intrasound therapy (LITR and HITR, respectively) given once daily and twice daily on the morphology and oxidative stress in healing tendon tissue following an acute injury.

Methods: Eighty-five male rats, randomized into six groups were further subdivided into groups A, B, and C, except for Group 1 which was subdivided into A and B only. Groups 2-6 underwent an induced crush injury. The six groups were allocated to: serve as controls (Group 1), receive no treatment (Group 2), HITR twice daily (Group 3), HITR once daily (Group 4), LITR twice daily (Group 5), and LITR once daily (Group 6). Intrasound therapy (ITR) was commenced 24 hours postinjury and was given once daily or twice daily over the first 14 days postinjury. The animals in subgroups A and B were sacrificed on day 15 postinjury, and those in subgroup $\mathrm{C}$ were sacrificed on day 31 postinjury. The tendons were excised, and processed for histology and malondialdehyde (MDA) assay.

Results: There was no significant difference in the tenocyte population between the HITR-and LITR-treated groups. However, twice-daily treatment in either the low- or high-intensity mode resulted in significant tenocyte proliferation compared with the once-daily treated groups, and also had the highest percentage of tenoblasts compared with the population of tenocytes in the proliferative phase of healing. All treatment protocols marginally lowered the MDA level.

Conclusion: The role of IRT in tendon healing is influenced more by the frequency of treatment rather than the intensity of the delivered dosage.

Keywords: intrasound therapy, tenoblast, tenocytes, tendon injury, crush injury, oxidative stress, malondialdehyde, tenoblast proliferation

\section{Introduction}

Tendon injuries are considered a major clinical challenge in orthopedic and sports medicine. ${ }^{1}$ Inadequate repair of acute tendon injuries and oxidative stress by reactive oxygen species (ROS) may precipitate tendinosis, which is characterized by tenocyte death, decreased matrix and collagen production, and increased risk of reinjury. ${ }^{2}$ Marsolais et $\mathrm{al}^{3}$ have reported that the poor healing response in tendons is due to an insufficient population of tenoblasts, and suggested investigations into other treatment modalities that may promote migration and activation of tenoblasts to influence tendon healing.

Two different types of specialized fibroblasts coexist in tendon, ie, elongated tenocytes which are morphologically distinct from ovoid-shaped tenoblasts, the latter being more involved in intrinsic healing. ${ }^{4}$ In the proliferative phase of healing, tenoblasts proliferate, lay down collagen fibers and, in the late phase of healing, become terminally differentiated into tenocytes. ${ }^{5}$ submit your manuscript $\mid$ www. dovepress.com

Dovepress

12118 
ROS, reported to be beneficial in healing at low physiologic levels by stimulating fibroblast proliferation are, however, toxic in large doses, resulting in lipid peroxidation and apoptosis, which can be monitored by tissue levels of oxidative stress markers like malondialdehyde (MDA) ${ }^{6-8}$ Fibroblasts also specifically generate ROS through an nicotinamide adenine dinucleotide phosphate oxidase complex in response to cytokines and growth factors, the production and release of which are stimulated after tendon injury. ${ }^{9,10}$

Sound waves, which are mechanical energy transmitted from one molecule to another, have been reported to interact with biologic tissue and promote healing following injury. ${ }^{11}$ Therapeutic ultrasound (TUS) which has been widely used in the treatment of soft tissue injuries, emits sound waves of high frequency between $1-3 \mathrm{mHz}$, which is above the threshold for human hearing. ${ }^{12}$ The intrasound vibrator massager, on the other hand, produces mixed frequency acoustic waves in the intrasonic range $(16-20,000 \mathrm{~Hz})$ and has been claimed to be effective in the management of a wide range of ailments, including improved healing in acute inflammatory injuries. ${ }^{13}$ However, the claims of efficacy are subjective accounts only because there are no relevant reported experimental or clinical trials. Intrasound is acclaimed by its manufacturers to be effective at any comfortable intensity and recommended to be used regularly for five minutes 2-3 times a day for best effects. This may be responsible for the design of the intrasound device, in which the exact delivered dosage is noncalibrated, and intensity is chosen over a wide range from low to high by adjusting the radial intensity knob anticlockwise. ${ }^{14}$ However, it may be ambiguous to expect the effects to be the same at any desired intensity. Our pioneer research suggested that both low and high intensity IRT caused a proliferation of tenocytes and lowered oxidative stress when given on alternate days following an acute tendon injury, although low intensity resulted in significant proliferation. ${ }^{15}$ The purpose of this study was to determine and compare the effects of the delivered intensity of IRT with the frequency of treatment on tendon healing following an acute injury.

\section{Materials and methods \\ Materials}

Eighty-five 10-week-old male Sprague Dawley albino rats weighing 160-180 g were used for the study. The animals were kept in the animal room at the Department of Anatomy, College of Medicine, University of Lagos, where the study was carried out. They were kept under standard conditions of 12-hour light and 12-hour darkness photoperiodicity.
Ethical approval for the study was obtained from the Ethical Committee of the College of Medicine, University of Lagos, Nigeria. The rats were fed on commercial rat chow and water ad libitum. The Novasonic-Novafon (Denmark) intrasonic device was used in this study.

\section{Methods \\ Experimental protocol}

The animals were randomly assigned to one of six groups, with 15 rats per group (except for the control group which had 10 rats) as follows: Group 1 (normal control, nil injury, nil treatment); Group 2 (experimental control, injury, nil treatment); Group 3 (injury followed by high-intensity intrasound [HIRT] twice daily); Group 4 (injury followed by HIRT once daily); Group 5 (injury followed by low-intensity intrasound [LIRT] twice daily); and Group 6 (injury followed by LIRT once daily).

The rats in each group were further randomly subdivided into groups A, B, and C, with five rats in each subgroup. The animals in Group 1 were subdivided into groups A and B only. In subgroup A, tendons were processed for histology in the proliferative phase. In subgroup B, tendons were processed for MDA assay. In subgroup $C$, tendons were processed for histology in the remodelling phase.

\section{Injury procedure}

The preinjury circumference of the left hind limbs for each rat was measured $2 \mathrm{~cm}$ above the calcaneal insertion of the Achilles tendon by means of a flexible inextensible marked cord. The Achilles tendon of the left limb was clamped with Number 1 artery forceps to the maximum forceps closure for 60 seconds to induce a crush injury. ${ }^{16}$ Twenty-four hours postinjury, the postinjury circumference of the hind limb

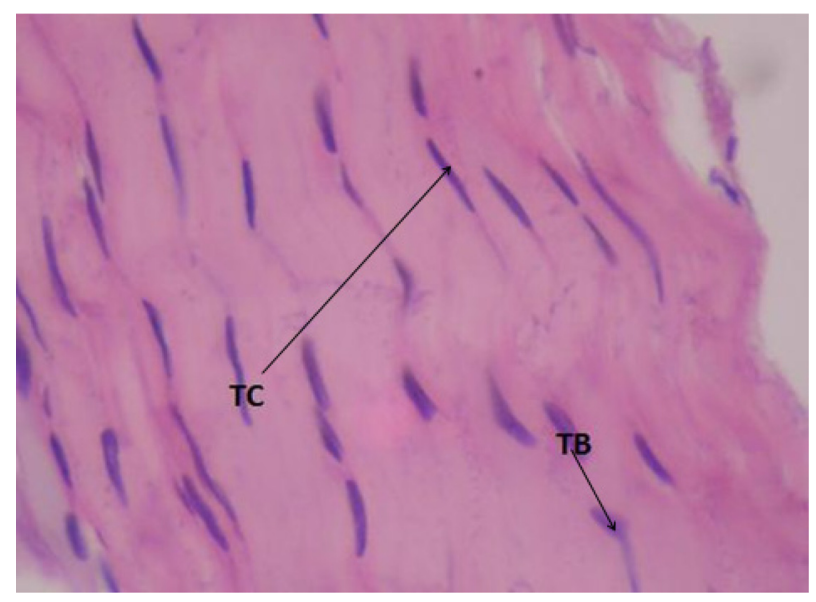

Figure I Group I L/S of tendon in the control group showing TC elongated tenocytes and TB ovoid tenoblasts (hematoxylin and eosin, 400x). 


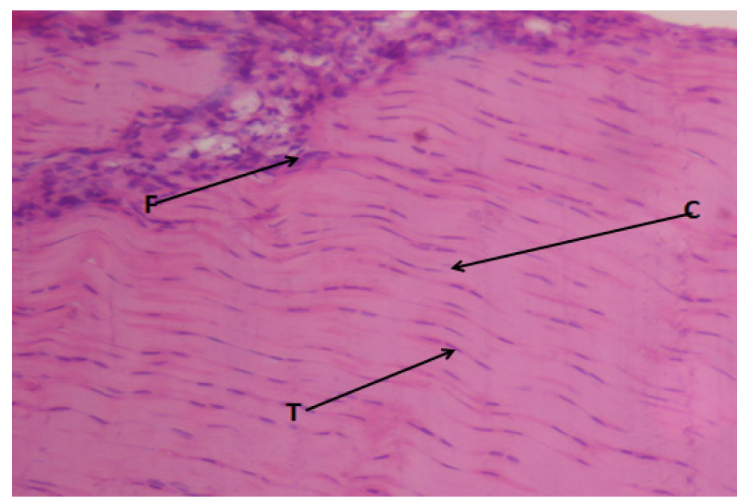

Figure 2A Group 2A: L/S of tendon of rat in the experimental control group in the early proliferative phase. $\mathbf{F}$ Infiltration of mononuclear inflammatory cells. $\mathbf{T}$ Proliferating immature tenocytes. C Relative absence of well-formed collagen fibers. (hematoxylin and eosin, I00X).

was taken again at the same marked point to ascertain the presence of edema, as a sign of inflammation.

\section{Intrasound therapy}

The Achilles tendon of the left hind limb of each of the rats was treated with either the lowest or highest intensity of IRT using the small treatment head applied over the Achilles tendon for five minutes and sterile $\mathrm{K}-\mathrm{Y}$ jelly as a coupling medium. ${ }^{17}$ Treatment was given for 14 days postinjury for six consecutive days with a one day interval between treatments. ${ }^{18}$ Each rat had IRT for 12 days. Intrasound was not administered on day 7 or on day 14 postinjury. The intrasound machine was factory-calibrated at low and high intensity only.

\section{Animal sacrifice and tendon harvest process}

On day 15 postinjury, the animals were sacrificed and the Achilles tendons excised.

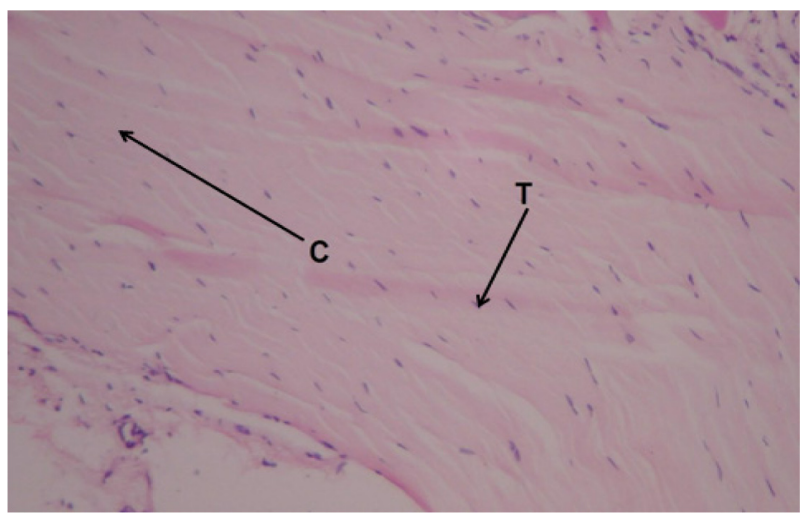

Figure 2B Group 2C: L/S of tendon of rat in the experimental control group in the remodeling phase. Showing $\mathbf{C}$ collagen fibers not well-aligned and $\mathbf{T}$ tenocytes that are randomly oriented (hematoxylin and eosin, I00X).

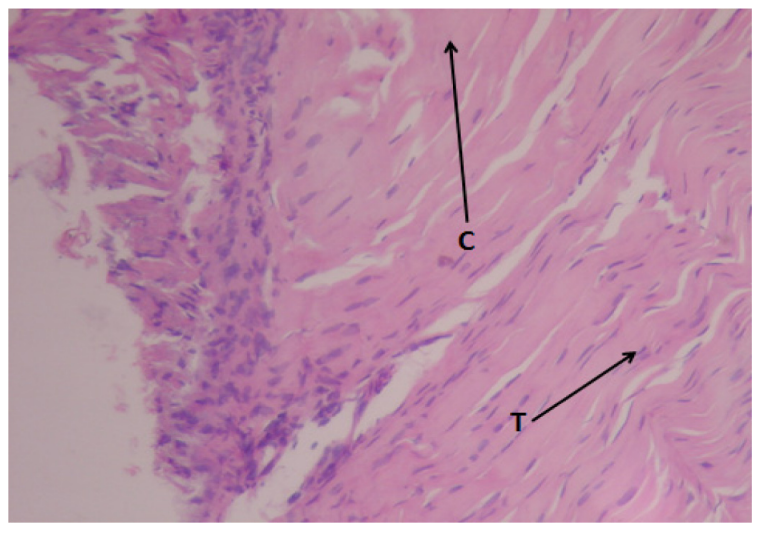

Figure 3A Group 3A: L/S of injured tendon treated with high-intensity intrasound twice daily in the proliferative phase. T Proliferating plump and mature tenocytes. C) Broad bands of dense, compact and well-aligned collagen fibers (hematoxylin and eosin, 100X).

The tendons in subgroup A were processed for histology using the stereologic technique described by Young and Dyson, ${ }^{12}$ while those in subgroup B were assayed for MDA. On day 31 postinjury, the animals in subgroup $C$ were sacrificed and the left Achilles tendon excised and processed for histologic studies.

\section{Stereologic analysis}

The slides were observed under the light microscope fitted with an ocular test grid at a magnification of $100 \times$ and 400x using the method of Cruz-Orive and Weibel. ${ }^{19}$ The tenoblast and tenocyte profiles identified were the nucleus. Fifty random values (10 per animal) were obtained for each group. The numerical density represents the number of tenocyte profiles per unit area of field. ${ }^{20}$ This is estimated as the profile number of tenocytes $(\mathrm{N})$ within the frame of the test grid (A). $\mathrm{N}$ was determined by counting all the tenocyte profiles partially or totally

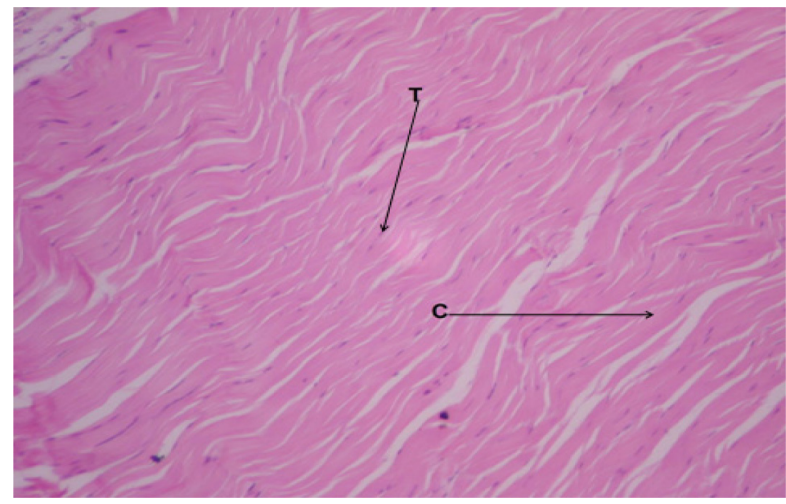

Figure 3B Group 3C: L/S of injured tendon treated with high-intensity intrasound twice daily in the remodeling phase showing $\mathbf{T}$ scanty cells and $\mathbf{C}$ well-aligned collagen fibers (hematoxylin and eosin, I00X). 


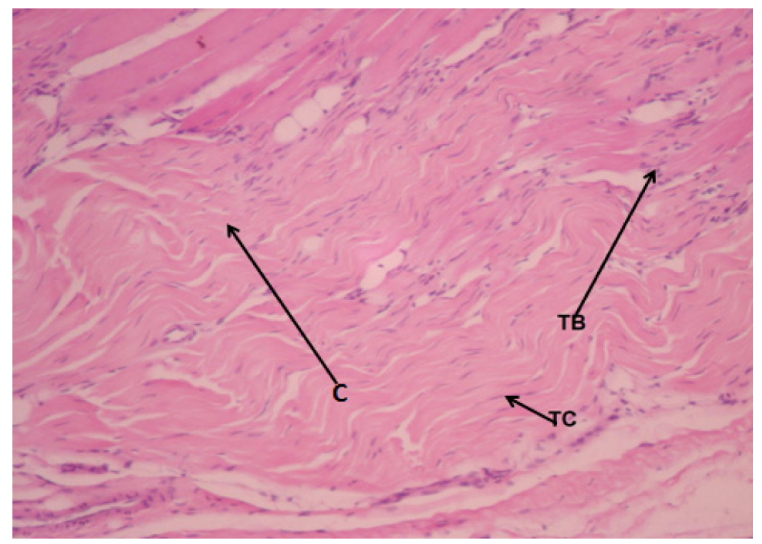

Figure 4A Group 4A: L/S of injured tendon treated with high-intensity intrasound once daily in the proliferative phase showing $\mathbf{C}$ new collagen fibers and a mixture of tenoblasts and tenocytes (hematoxylin and eosin, I00X).

within the frame area that did not intersect the forbidden lines, which are the top and left margins of the test grid. ${ }^{19}$ Tenoblasts were further differentiated from the typical tenocytes by their shapes. The elongated cells were identified as tenocytes, while the ovoid cells were identified as tenoblasts. ${ }^{21} 150$ random values ( 30 per animal) were obtained for each group.

\section{Statistical analysis}

The statistical software used was the EPI-INFO 3.5.12008 version. The data obtained from the stereologic evaluation of the combined tenoblast and tenocyte populations are expressed as the mean \pm standard deviation (SD). Differences between the groups were compared using analysis of variance (ANOVA). In order to eliminate the possibility of nonsignificant values masking the effect of significant values as a result of the large sample sizes, paired samples were further analysed using the Student's $t$-test for analysis of paired

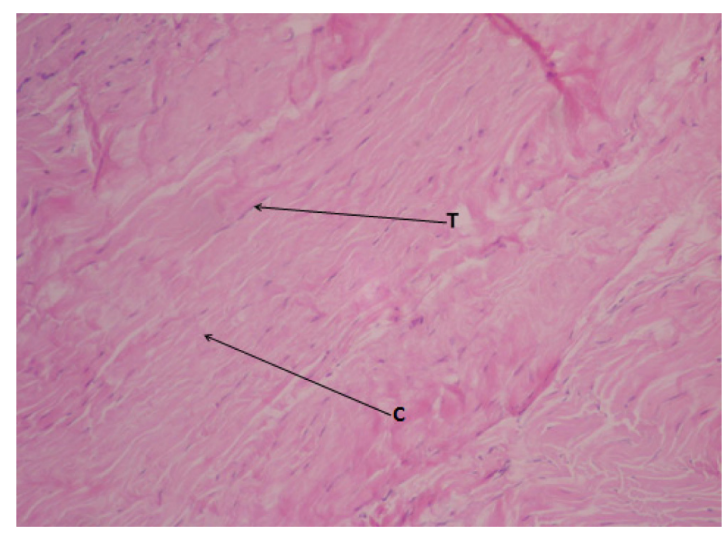

Figure 4B Group 4C: L/S of injured tendon treated with high-intensity intrasound once daily in the remodeling phase. $T$ tenocytes and $C$ well-aligned collagen fibers (hematoxylin and eosin, I00X).

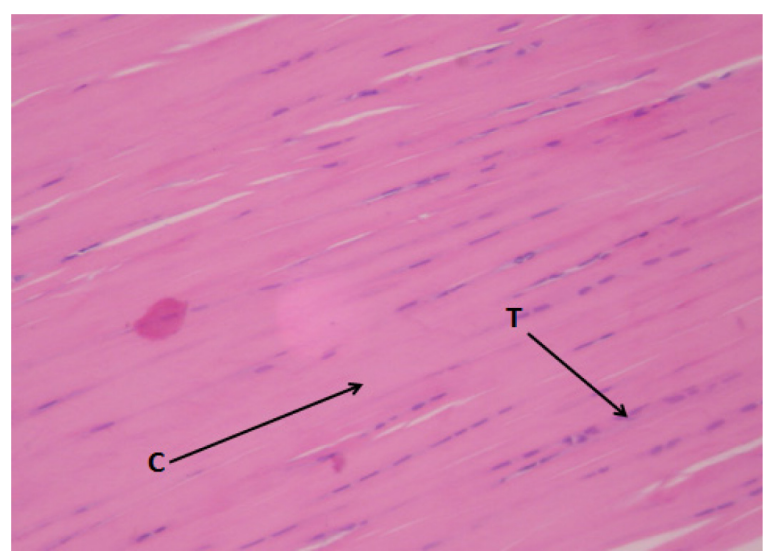

Figure 5A Group 5A: L/S of injured tendon in the proliferative phase treated with low-intensity intrasound twice daily showing $\mathbf{T}$ tenocytes and $\mathbf{C}$ new collagen fibers (hematoxylin and eosin, I00X).

samples. The data were also expressed as percentages and the groups compared. The null hypothesis was set at a significant level of 0.05 or $5 \%$.

\section{Results}

The twice-daily IRT modalities resulted in a significant $(P<0.05)$ increase in the overall population of tenocytes when compared with the control group, unlike the daily treatment which marginally increased the tenocyte population (Table 1).

The combined tenocyte population for high-intensity once daily and twice daily IRT groups was compared with the combined population of the low-intensity once daily and twice daily IRT groups. Results show that there is no significant difference between the combined groups. On the contrary, when the tenocyte population for the combined low- and high-daily IRT group was compared with the combined population of the low and high twice-daily IRT

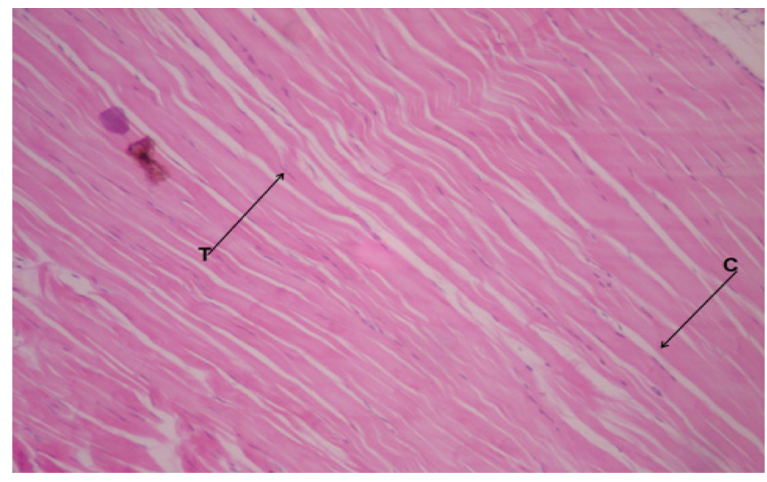

Figure 5B Group 5C: L/S of injured tendon treated with low-intensity intrasound twice daily in the remodeling phase showing $C$ well-aligned collagen fibers and $T$ axially aligned tenocytes (hematoxylin and eosin, I00X). 


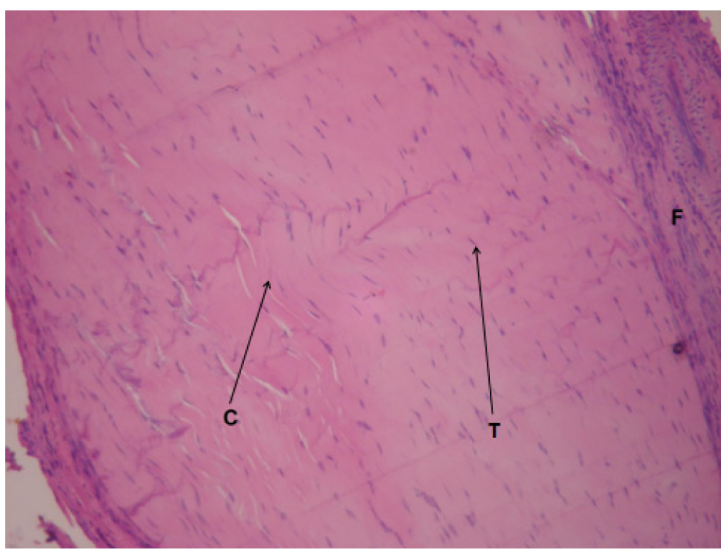

Figure 6A Group 6A: L/S of injured tendon treated with low-intensity intrasound once daily in the proliferative phase showing $\mathbf{C}$ new collagen fibers, $\mathbf{F}$ inflammatory cells and $\mathbf{T}$ tenocytes (hematoxylin and eosin, I00X).

group, there was a significant $(P<0.05)$ increase in the population of the tenocytes in the combined twice-daily groups (Table 1).

Table 2 shows that the IRT twice daily groups had the highest percentage of tenoblasts compared with the population of tenocytes in the proliferative phase of healing, while there was a sharp reduction in the population of tenoblasts, with a corresponding increase in tenocyte population at 30 days compared with values in the proliferative phase.

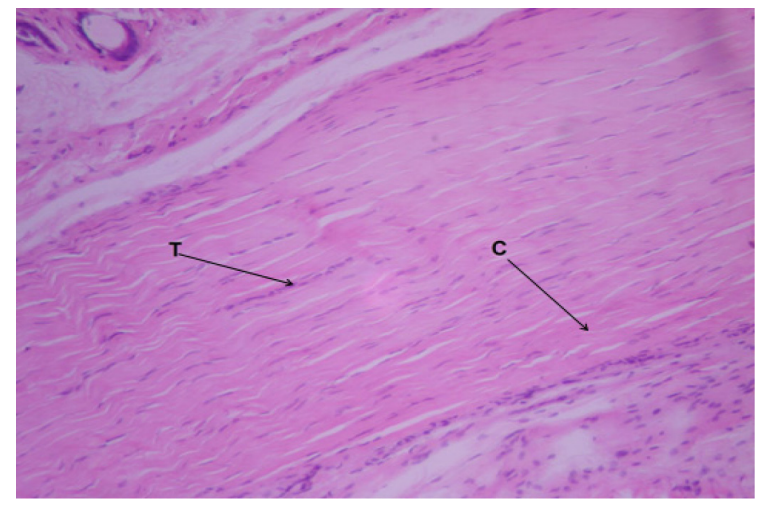

Figure 6B Group 6C: L/S of injured tendon treated with low-intensity intrasound once daily in the remodeling phase. $\mathbf{T}$ axially aligned tenocytes and $\mathbf{C}$ well-aligned collagen fibers (hematoxylin and eosin, I00X).

All treatment protocols marginally lowered the MDA level, except for the HITR once daily treatment, which resulted in a significant $(P=0.05)$ reduction (Table 3$)$.

Compared with the tendon that was injured and allowed to heal without any therapeutic intervention (Group 2A; Figure 2A) which showed massive inflammation and a relative absence of well-formed collagen fibers at 14 days, all the IRT-treated tendons showed mild inflammation and well-aligned collagen fibers (Groups 3-6A; Figures 3A-6B). In the late phase of healing, compared with the normal tendon (Group 1; Figure 1), all the

Table I Group comparison of tenocyte population between the experimental groups

\begin{tabular}{|c|c|c|}
\hline Comparison of tenocyte counts between Groups 2 and 3 & Group 2 & Group 3 \\
\hline Mean tenocyte count & $20.7 \pm 4.3$ & $27.3 \pm 9.2$ \\
\hline \multicolumn{3}{|l|}{$P$ value $=0.03^{*}$} \\
\hline Comparison of tenocyte counts between Groups 2 and 4 & Group 2 & Group 4 \\
\hline $\begin{array}{l}\text { Mean tenocyte count } \\
P \text { value }=0.06\end{array}$ & $20.7 \pm 4.3$ & $23.1 \pm 4.6$ \\
\hline Comparison of tenocyte counts between Groups 2 and 5 & Group 2 & Group 5 \\
\hline Mean tenocyte count & $20.7 \pm 4.3$ & $28.2 \pm 9.8$ \\
\hline \multicolumn{3}{|l|}{$P$ value $=0.04^{*}$} \\
\hline Comparison of tenocyte counts between Groups 2 and 6 & Group 2 & Group 6 \\
\hline Mean tenocyte count & $20.7 \pm 4.3$ & $25.1 \pm 6.9$ \\
\hline \multicolumn{3}{|l|}{$P$ value $=0.05^{*}$} \\
\hline Comparison of tenocyte counts between Groups 3 and 5 & Group 3 & Group 5 \\
\hline Mean tenocyte count & $27.3 \pm 9.2$ & $28.2 \pm 9.8$ \\
\hline \multicolumn{3}{|l|}{$P$ value $=0.07$} \\
\hline Comparison of tenocyte counts between Groups 4 and 6 & Group 4 & Group 6 \\
\hline Mean tenocyte count & $23.1 \pm 4.6$ & $25.1 \pm 6.9$ \\
\hline \multicolumn{3}{|l|}{$P$ value $=0.09$} \\
\hline Comparison of tenocyte counts between Groups 3, 4, 5 and 6 & Groups 3 and 4 & Groups 5 and 6 \\
\hline Mean tenocyte count & $25.2 \pm 5.6$ & $26.6 \pm 6.8$ \\
\hline \multicolumn{3}{|l|}{$P$ value $=0.29$} \\
\hline Comparison of tenocyte counts between Groups 3, 5, 4 and 6 & Groups 3 and 5 & Groups 4 and 6 \\
\hline $\begin{array}{l}\text { Mean tenocyte count } \\
P \text { value }=0.04^{*}\end{array}$ & $27.8 \pm 6.6$ & $24.1 \pm 10.9$ \\
\hline
\end{tabular}

Key: Group 2, injury, nil treatment; Group 3, high-intensity intrasound twice daily; Group 4, high-intensity intrasound once daily; Group 5 , low-intensity intrasound twice daily; Group 6, low-intensity intrasound once daily. *Significant at $P<0.05$. 
Table 2 Comparison of the percentage of tenoblasts and tenocytes in the experimental groups between day 14 and day 30 of treatment

\begin{tabular}{|c|c|c|c|c|c|c|c|c|c|c|c|c|}
\hline Groups & I & & 2 & & 3 & & 4 & & 5 & & 6 & \\
\hline Cells (\%) & TB & TC & TB & TC & TB & TC & TB & TC & TB & TC & TB & TC \\
\hline Day 14 & 22.8 & 77.2 & 32.3 & 67.7 & 49.6 & 50.4 & 37.9 & 62.1 & 40.5 & 59.9 & 26.4 & 73.6 \\
\hline Day 30 & & & 31.0 & 69.0 & 24.1 & 75.9 & 34.0 & 66.0 & 14.3 & 85.7 & 23.8 & 76.2 \\
\hline
\end{tabular}

Key: Group I, nil injury (control); Group 2, injury, nil treatment; Group 3, high-intensity intrasound twice daily; Group 4, high-intensity intrasound once daily; Group 5, low-intensity intrasound twice daily; Group 6, low-intensity intrasound once daily.

Abbreviations: TB, tenoblasts; TC, tenocytes.

IRT-treated tendons showed bundles of well-aligned collagen fibers with a paucity of cells, especially the twice-daily treated tendons (Groups 3C and 5C; Figures 3B and 5B respectively).

\section{Discussion}

The management of tendon injuries has remained a major challenge in sports and occupational medicine. This study was undertaken as part of an ongoing attempt to investigate some physical modalities that have been claimed to promote the healing of soft tissue injuries, although most of these claims are subjective accounts. One of these modalities is IRT which is becoming popular in clinical practice. The aim of this study was to establish the scientific basis for the acclaimed positive effects of IRT at any desired intensity in the healing of acute tendon injuries. The manufacturer of the Novasonic intrasound device had suggested in the literature that treatment be administered 2-3 times daily at any comfortable intensity. ${ }^{13}$ This is quite pertinent because the delivered intensity is not calibrated on the machine.

Tenoblasts, the activated form of tenocytes, play a key role in tendon repair because they lay down the extracellular matrix, and the strength of the repaired tissue has been attributed to their population at the site of injury. ${ }^{22}$ Treatment modalities that stimulate tenoblast proliferation will enhance tendon healing. ${ }^{23}$ When ITR is administered once daily, both the low and high intensities are equally effective in causing tenoblast proliferation because there is no significant difference between their tenocyte populations (Table 1). However, when compared with the twice daily groups, the latter had better therapeutic effects by inducing a significant proliferation of tenoblasts. In comparing the lowand high-intensity twice daily treatment groups, there was no significant difference between the tenocyte populations (Table 1) and both had positive effects on the morphology of the healing tendon, with well laid out collagen fibers and proliferation of tenoblasts at 14 days postinjury (Groups 3 and 5) compared with the injured untreated group
(Group 2). This appears to be in contrast with the results of an earlier study by these authors in which concurrent administration of indomethacin daily and alternate-day treatment with IRT shows that the high intensity appeared to ameliorate the deleterious effects of this drug on the healing tendon by causing a proliferation of tenoblasts while the drug further repressed the proliferation of tenoblasts when combined with low-intensity IRT. ${ }^{24}$

Since the proportion of tenoblasts to tenocytes following injury is an indicator of tissue response to treatment given, ${ }^{25}$ these findings indicate that IRT does stimulate proliferation of tenoblasts in the early phase of healing especially when applied twice daily. In the late phase of healing, compared

Table 3 Effect of once-daily and twice-daily treatment with intrasound therapy on malondialdehyde expression

\begin{tabular}{|c|c|c|}
\hline & Group 2 & Group 3 \\
\hline $\begin{array}{l}\text { Comparison of MDA level } \\
\text { between Groups } 2 \text { and } 3 \\
\text { Mean MDA level } \\
P \text { value }=0.92\end{array}$ & $0.99 \pm 0.04$ & $0.91 \pm 0.04$ \\
\hline $\begin{array}{l}\text { Comparison of MDA level between } \\
\text { Groups } 2 \text { and } 4\end{array}$ & Group 2 & Group 4 \\
\hline $\begin{array}{l}\text { Mean MDA level } \\
P \text { value }=0.53\end{array}$ & $0.99 \pm 0.04$ & $0.61 \pm 0.03$ \\
\hline $\begin{array}{l}\text { Comparison of MDA level } \\
\text { between Groups } 2 \text { and } 5\end{array}$ & Group 2 & Group 5 \\
\hline $\begin{array}{l}\text { Mean MDA level } \\
P \text { value }=0.06\end{array}$ & $0.99 \pm 0.04$ & $0.97 \pm 0.04$ \\
\hline $\begin{array}{l}\text { Comparison of MDA level } \\
\text { between Groups } 2 \text { and } 6\end{array}$ & Group 2 & Group 6 \\
\hline $\begin{array}{l}\text { Mean MDA level } \\
P \text { value }=0.29\end{array}$ & $0.99 \pm 0.04$ & $0.76 \pm 0.03$ \\
\hline $\begin{array}{l}\text { Comparison of MDA level between } \\
\text { Groups } 2 \text { and Groups } 3 \text { and } 5\end{array}$ & Group 2 & Groups 3 and 5 \\
\hline $\begin{array}{l}\text { Mean MDA level } \\
P \text { value }=0.08\end{array}$ & $0.99 \pm 0.04$ & $0.94 \pm 0.01$ \\
\hline $\begin{array}{l}\text { Comparison of MDA level between } \\
\text { Groups } 2 \text { and Groups } 4 \text { and } 6\end{array}$ & Group 2 & Groups 4 and 6 \\
\hline $\begin{array}{l}\text { Mean MDA level } \\
P \text { value }=0.05^{*}\end{array}$ & $0.99 \pm 0.04$ & $0.68 \pm 0.02$ \\
\hline
\end{tabular}

Key: Group 2, injury, nil treatment; Group 3, high-intensity intrasound twice daily; Group 4, high-intensity intrasound once daily; Group 5, low-intensity intrasound twice daily; Group 6, low-intensity intrasound once daily. *Significant at $P<0.05$. Abbreviation: MDA, malondialdehyde. 
with injured untreated tendons, the twice daily treated tendons had a marked reduction in tenoblast population by 30 days when compared with values at 14 days. The population of tenoblasts in these groups at 14 days was about thrice and twice their population at 30 days for the low and high intensities, respectively. The daily treated tendons on the other hand had a marginal reduction in tenoblast population at 30 days (Table 2). These findings are pertinent because the principal tendon structure is reported to be restored by the fourth week postinjury with a terminal differentiation of most of the tenoblasts to tenocytes..$^{21,26}$

In addition, compared with the injured untreated and daily treated tendons, the histology of the tendons treated twice daily showed better aligned collagen fibres with scanty axially aligned tenocytes at 30 days (Groups 2-6).

Table 1 also shows that when the combined overall tenocyte population in the HITR once daily and twice daily groups was compared with that of the LITR once daily and twice daily, there was no significant difference. However, comparing the combined population of the low- and high-intensity once daily groups with the low- and high-intensity twice daily groups showed a significant $(P<0.05)$ difference in favor of the twice daily treated tendons. These findings suggest that the intensity of the delivered dosage of ITR may not play as great a role in its therapeutic effect on the healing tendon as the frequency of treatment.

All the ITR treatment protocols lowered the MDA values at 14 days postinjury. However, the once daily treatments had much lower values than the twice daily treatments, and the HITR once daily treatment resulted in a significant $(P=0.05)$ reduction in the MDA value compared with the control (Table 3 ). This is quite pertinent because only this group did not have a significant proliferation of tenocytes among all the experimental groups (Table 1). In addition, this group had a higher percentage of tenoblasts at day 30 postinjury compared with controls (Table 2). It does appear from the results of this study that MDA levels increase with frequency of treatment and, because the twice daily treatments had a better effect on the healing tendon, this finding suggests a role for ITR and some ROS, especially nitric oxide, in influencing tendon healing. ${ }^{10,27}$

These findings have shown that IRT is effective in tendon healing at any given intensity when applied twice daily. Therefore, this therapeutic device may be a simpler option to consider in the management of acute tendon injuries because complicated choices of treatment parameters which are common with most physical therapy modalities are eliminated. However, further studies are needed on the mechanism of action of this novel device especially as regards its interaction with ROS.

In conclusion, the role of IRT in tendon healing is influenced more by the frequency of treatment. The intensity of the delivered dosage plays a minimal role in tendon healing. IRT increases MDA expression in a frequencydependent manner. There is a dearth of scientific data on the role of IRT in tendon healing, so there are no prior studies for comparison. In addition, the exact delivered intensity cannot be ascertained, because the device is factory-calibrated into low- and high-intensity only.

\section{Disclosure}

The authors declare no conflicts of financial interest in this research.

\section{References}

1. Aslan H, Kimelman-Bleich N, Pelled G, Gazit D. Molecular targets for tendon neoformation. J Clin Invest. 2008;118:439-444.

2. Paoloni JA, Murrell GA. Three-year follow up study of topical glyceryl trinitrate treatment of chronic non insertional Achilles tendinopathy. Foot Ankle Int. 2007;28:1064-1068.

3. Marsolais D, Cote CH, Frenette J. Nonsteroidal anti-inflammatory drug reduces neutrophil and macrophage accumulation but does not improve tendon regeneration. Lab Invest. 2003;83:991-999.

4. Bagnaninchi PO, Yang Y, El Haj AJ, Maffulli N. Tissue engineering for tendon repair. Br J Sports Med. 2007;41:e10.

5. Sharma P, Maffulli N. Tendon injury and tendinopathy: Healing and repair. J Bone Joint Surg Am. 2005;87:187-202.

6. Buege JA, Aust SD. Microsomal lipid peroxidation In: Fleischer S, Packer L, editors. Methods in Enzymology. New York, NY: Academic Press; 1978.

7. Murrell GAC, Francis MJO, Bromley L. Modulation of fibroblast proliferation by oxygen free radicals. Biochem J. 1990;26:659-665.

8. Murrell GA, Szabo C, Hannafin JA, et al. Modulation of tendon healing by nitric oxide. Inflamm Res. 1997;46:19-27.

9. Meier B, Radeke HH, Selle S. Human fibroblasts release reactive oxygen species in response to interleukin-1 or tumour necrosis factor-alpha. Biochem J. 1989;263:539-545.

10. Banes AJ, Tsuzaki M, Hu P. PDGF-BB, IFF-I and mechanical load stimulate DNA synthesis in avian tendon fibroblasts in vitro. J Biomech. 1995;28:1505-1513.

11. Aluclu I, Dalgic A, Toprak ZF. A fuzzy logic-based model for noise control at industrial workplaces. Appl Ergon. 2008;39:368-378.

12. Young SR, Dyson M. The effect of therapeutic ultrasound on the healing of full thickness excised skin lesions. Ultrasound. 1990;28:175-180.

13. Dancocks A, Rouse A, Hiscox J. A pilot study to assess the sensitivity and specificity of an intrasound device in the diagnosis of ankle fracture. J Accid Emerg Med. 1997;14:230-232.

14. Novasonic. How Novasonic-Novafon massager works. Available at: http:// www.novasonicwest.com/novasonic.asp. Accessed on June 06, 2007.

15. Akinbo SR, Aiyegbusi AI, Duru FIO, Noronha CC, Okanlawon AO. The efficacy of intrasound therapy on the acute tendon injury. The Internet Journal of Orthopedic Surgery. 2009;13:2.

16. de Tarso P, de Carvalho C, Schettert Silva I, et al. Histological study of tendon healing in malnourished Wistar rats treated with ultrasound therapy. Acta Cir Bras. 2006;21 Suppl 4:13-17.

17. Potawski L, Watson T. Relative transmissivity of ultrasound coupling agents commonly used by therapist in the UK. Ultrasound Med Biol. 2007;33:120-128. 
18. Fu SC, Shum WT, Hung LK, Wong MW, Qin L, Chan KM. Low-intensity pulsed ultrasound on tendon healing: A study of the effect of treatment duration and treatment initiation. Am J Sports Med. 2008;36:1742-1749.

19. Cruz-Orive LM, Weibel ER. Recent stereological methods for cell biology brief survey. Am J Physiol. 1990;258(4 Pt 1):L148-L156.

20. Gundersen HJG. Stereology of arbitrary particle: A review of unbiased number and size estimators and the presentation of new ones, in memory of William R Thompson. J Microsc. 1986;143(Pt 1):3-45.

21. Chuen FS, Chuk CY, Ping WY, Nar WW, Kim HL, Ming CK. Immunohistochemical characterization of cells in adult human patellar tendons. J Histochem Cytochem. 2004;52:1151-1157.

22. Alexander S, Khan KM, Cook JL, Duronio V. Human tendon overuse pathology: Histopathologic and biochemical findings. In: Woo S, Renström P, Arnoczky SP, editors. Tendinopathy in Athletes. Hoboken, NJ: Wiley-Blackwell; 2008.
23. Caring Medical. NSAIDs: Why we do not recommend them. Sports Injury Med. 2007;97:472-476.

24. Aiyegbusi AI, Akinbo SR, Noronha CC, Okanlawon AO. The effects of varied intensities of intrasound therapy with indomethacin on the morphology of the healing tendon. Nig $Q J$ Hosp Med. 2010;20: 19-23.

25. Davidson CJ, Ganion LR, Gehlsen GM, Verhoestra B, Roepke JE, Sevier TL. Rat tendon morphologic and functional changes resulting from soft tissue mobilization. Med Sci Sports Exerc. 1997;29: 313-319.

26. Yuko A, Tomoyuki K, Seigo U, Minoru H, Seiichi K. Repair process of the rat Achilles tendon after tenotomy. J Health Sci. 2006;6:32-42.

27. Sundaresan M, Yu Z-X, Ferrans VJ. Requirement for generation of $\mathrm{H}_{2} \mathrm{O}_{2}$ for platelet derived growth factor signal transduction. Science. 1995;270:296-299.

\section{Publish your work in this journal}

Open Access Rheumatology Research and Reviews is an international, peer-reviewed, open access journal, publishing all aspects of clinical and experimental rheumatology in the clinic and laboratory including the following topics: Pathology, pathophysiology of rheumatological diseases; Investigation, treatment and management of rheumatological diseases; Clinical trials and novel pharmacological approaches for the treatment of rheumatological disorders. The manuscript management system is completely online and includes a very quick and fair peerreview system, which is all easy to use. Visit http://www.dovepress.com/ testimonials.php to read real quotes from published authors. 